\title{
Classificação de Estados Epilépticos em Sinais de EEG Utilizando Deteç̧ão de Anomalias
}

\author{
Lucas Cabral $^{1}$, Guilherme A. Barreto ${ }^{2}$, José Maria Monteiro ${ }^{1}$ \\ ${ }^{1}$ Departamento de Computação - Universidade Federal do Ceará (UFC) \\ Fortaleza, CE - Brazil \\ ${ }^{2}$ Departamento de Engenharia de Teleinformática - Universidade Federal do Ceará (UFC) \\ Fortaleza - CE - Brazil \\ lucascccealu.ufc.br, gbarretodufc.br, monteiro@dc.ufc.br
}

\begin{abstract}
Epilepsy is a neurological disorder characterized by an abnormal electrical disturbance in the brain, causing recurrent seizures. The most commonly used exam to diagnose epilepsy it the electroencephalogram (EEG), where a patient's brain electrical activity is measured and visually analyzed. However, identifying epileptic patterns in the EEG signal through visual inspection is a time-consuming and exhaustive task for professionals in the field, motivating the development of algorithms that can identify these patterns, aiding the medical diagnosis. In this work, we propose three models based on anomaly detection. The results obtained demonstrate high performance and noise robustness in relation to results found in the literature.
\end{abstract}

Resumo. A epilepsia é um distúrbio neurológico caracterizado por uma perturbação elétrica anormal no cérebro, causando convulsões recorrentes. $O$ exame mais utilizado no diagnóstico da epilepsia é eletroencefalograma (EEG), onde a atividade elétrica cerebral de um paciente é mensurada e analisada visualmente. Contudo, identificar os padrões epilépticos no sinal de EEG através de inspeção visual é uma tarefa demorada e exaustiva para profissionais da área. Assim, o desenvolvimento de algoritmos que possam identificar esses padrões de forma automática, auxiliando o diagnóstico médico, tornou-se um importante desafio. Neste trabalho, propomos três modelos de classificação, baseados em detecção de anomalias. Os resultados obtidos demonstram alto desempenho e robustez a ruídos em relação resultados encontrados na literatura.

\section{Introdução}

A epilepsia é uma desordem crônica do cérebro que afeta cerca de 50 milhões de pessoas ao redor do mundo, sendo um dos distúrbios neurológicos mais frequentes [World Health Organization 2017]. Ela é caracterizada por crises recorrentes onde ocorre uma disfunção temporária da atividade elétrica dos neurônios, onde os sintomas de cada crise dependerão das partes do cérebro envolvidas na disfunção [Kanashiro 2006]. A crise convulsiva é a forma mais conhecida e é identificada comumente como "ataque epiléptico". Nesse tipo de crise, o portador de epilepsia pode cair ao chão, apresentar contrações musculares em todo o corpo, perda de consciência, mordedura da língua, salivação intensa, respiração ofegante, perda do controle da função intestinal e da bexiga. 
Portadores de epilepsia tendem a ter risco sofrer fraturas e contusões de lesões relacionadas a convulsões, bem como taxas mais altas de condições psicológicas como ansiedade e depressão. Tais pacientes apresentam um risco de morte prematura cerca de três vezes maior que a população em geral.

Uma importante ferramenta para o diagnóstico e a investigação da epilepsia é o eletroencefalograma (EEG) de escalpo, que consiste no registro da atividade elétrica cerebral captada por eletrodos posicionados no couro cabeludo. O EEG permite visualizar os comportamentos anormais característicos dos diversos tipos de crises e síndromes epilépticas, sendo o método mais específico para o diagnóstico das epilepsias [Niedermeyer and da Silva 2001]. Embora técnicas mais recentes de neuroimagem tenham trazido grandes avanços, o EEG continua sendo bastante utilizado, devido a sua simplicidade técnica e ao seu baixo custo.

Contudo, a atividade cerebral do ser humano apresenta uma infinidade de padrões de atividade, caracterizados pela soma de diferentes estados mentais em um instante de tempo, resultando em um sinal naturalmente complexo e não-linear [Adeli and Ghosh-Dastidar 2010]. Ademais, os exames de EEG geram uma enorme quantidade de dados, onde a duração de uma crise convulsiva é curta em relação a duração total do exame. Em exames com horas de duração os períodos de crise podem durar apenas alguns minutos ou segundos. Neurologistas precisam analisar centenas de horas de exame buscando esses padrões. Assim, o diagnóstico da epilepsia é um procedimento lento e sujeito a falhas, devido à fadiga visual e à subjetividade de cada especialista.

Neste contexto, algoritmos que classifiquem de forma automática os padrões relacionados à uma crise convulsiva são extremamente importantes para auxiliar o diagnóstico da epilepsia e facilitar o trabalho dos neurologistas. Porém, para que os algoritmos de classificação por aprendizado supervisionado apresentem um bom desempenho, o número de exemplos por classe deve ser balanceado [Webb 2002]. No caso dos sinais de EEG, existe um grande desbalanceamento entre a quantidade de dados referentes a crises convulsivas e dados associados a comportamentos não-convulsivos. Essas condições motivam o uso de técnicas de detecção de anomalias por aprendizado não-supervisionado, com a finalidade de classificar sinais EEG em convulsivo e não-convulsivo.

Neste trabalho, propomos e avaliamos o desempenho de três modelos de classificação de estados convulsivos em EEG baseados na detecção de anomalias e utilizando atributos no domínio da frequência de Densidade Espectral de Potência (PSD). Os resultados mostram um desempenho semelhante aos melhores resultados encontrados na literatura, possuindo robustez a sinais ruidosos, além de simplicidade de treino e baixo custo computacional.

\section{Trabalhos Relacionados}

São encontradas na literatura diferentes técnicas de classificação utilizando aprendizado supervisionado e diferentes métodos de extração de atributos com o objetivo de classificar corretamente os sinais de EEG em convulsivo e não-convulsivo. Não existe um método padrão de extração de atributos para sinais de EEG, e a combinação efetiva de atributos e classificador ainda é um problema em aberto. A maioria dos trabalhos encontrados na literatura é paciente-específico, ou seja, os modelos são treinados e testados com dados de um paciente por vez, devido a alta variabilidade de atividade cerebral en- 
tre pacientes. Subasi and Ercebeli 2005 obtiveram taxas de acerto em torno de 89,3 à 93\% utilizando redes neurais artificiais e regressão logística. Chan et al. 2008 apresentaram uma sensibilidade na classificação em torno de 84,89 à 94\% utilizando a extração de atributos no domínio da frequência e o classificador do tipo Support Vector Machine (SVM). Khan et al. 2012 usaram extração de atributos com Wavelet, produzindo acurácia de $91.8 \%$, sensibilidade de $83.6 \%$ e especificidade de 100\%. Acharya et al. 2011 utilizaram Recurrence Quantification Analysis (RQA) como método de extração de atributos e testou com sete diferentes classificadores: SVM, Gaussian Mixture Model (GMM), Fuzzy Sugeno Classifier, K-Nearest Neighbor (KNN), Naive Bayes Classifier (NBC), Decision Tree (DT), e Radial Basis Probabilistic Neural Network (RBPNN). Os melhores resultados foram obtidos com SVM, com acurácia de 96\%, sensibilidade de $96 \%$ e especificidade de $97 \%$. Shoeb and Guttag 2010 usaram decomposição Wavelet multi-nível para extrair atributos que capturam a morfologia e distribuição espacial do EEG, classificando com SVM e obtendo acurácia de $96 \%$.

Fergus et al. 2016 apresentaram um método para generalizar a detecção de crises convulsivas entre diferente pacientes, uma tarefe de maior dificuldade. Os vetores de atributos em seu trabalho foram gerados com os dados de 23 pacientes, usando Peak Frequency, Median Frequency, variância, root mean squares (RMS), sample entropy, assimetria e curtose. Foi apresentado um resultado de $88 \%$ de acurácia, $88 \%$ de sensibilidade e $93 \%$ de especificidade, utilizando um classificador KNN. Ainda considerando o problema de classificação inter-pacientes, Liang et al. 2019 apresentaram uma abordagem baseada em aprendizado profundo, utilizando uma combinação das redes neurais Convolutional Neural Network (CNN) e Long Short-Term Memory Network (LSTM), denominada Long-Term Convolutional Network (LTCN). Uma das vantagens da LTCN é o fato de não ser necessária uma etapa de manual de extração de atributos, pois esta trabalha diretamente com o sinal. Essa proposta apresentou acurácia de 84\%, sensibilidade de 99\% e especificidade de $99 \%$.

\section{Modelos Propostos}

A detecção de anomalias refere-se ao problema de encontrar dados que não estejam de acordo com o comportamento esperado. A detecção de anomalias encontra uso extensivo em uma ampla variedade de aplicações, como detecção de fraude para cartões de crédito, seguros ou cuidados de saúde, detecção de intrusão para cibersegurança, detecção de falhas em sistemas e diagnóstico de patologias [Varun Chandola 2009]. Em geral, em métodos de detecção de anomalias calcula-se uma medida de dissimilaridade entre os dados e as anomalias são encontradas através da comparação dessa medida com um limiar de decisão.

Neste trabalho, propomos três modelos de detecção de anomalias para detectar sinais convulsivos: Distâncias Ao Centroide Não-Convulsivo (DCNC), Distâncias Ao Centroide Convulsivo (DCC) e Distâncias aos Centroides de K-Clusters (DCKC).

\subsection{Modelo das Distâncias Ao Centroide Não-Convulsivo (DCNC)}

Uma medida de dissimilaridade bastante utilizada é a distância euclidiana ao centroide da massa de dados. Usualmente, um vetor com um valor alto para a distância ao centroide é considerado uma anomalia [Knorr 2000]. O valor do limiar de decisão usualmente é escolhido como o 95 ou 99 percentil das distâncias ao centroide [A. Zimek and Kriegel 2012]. 
O modelo das Distâncias Ao Centroide Não-Convulsivo utiliza apenas os dados não-convulsivos para definir o comportamento esperado, calculando o centroide da massa de dados não-convulsivos e calculando um vetor de distâncias de cada observação para o centroide. O limiar de decisão pode então ser escolhido como o 95 percentil desse vetor de distâncias. Esse modelo parte da suposição de que os dados possuem uma distribuição normal em torno do centroide, e pontos muitos distantes deste centroide são anomalias. Devido a alta dimensionalidade dos vetores, a visualização de sua distribuição espacial é complexa, sendo necessário testar a hipótese de sua distribuição normal. O Algoritmo 1 detalha esse método.

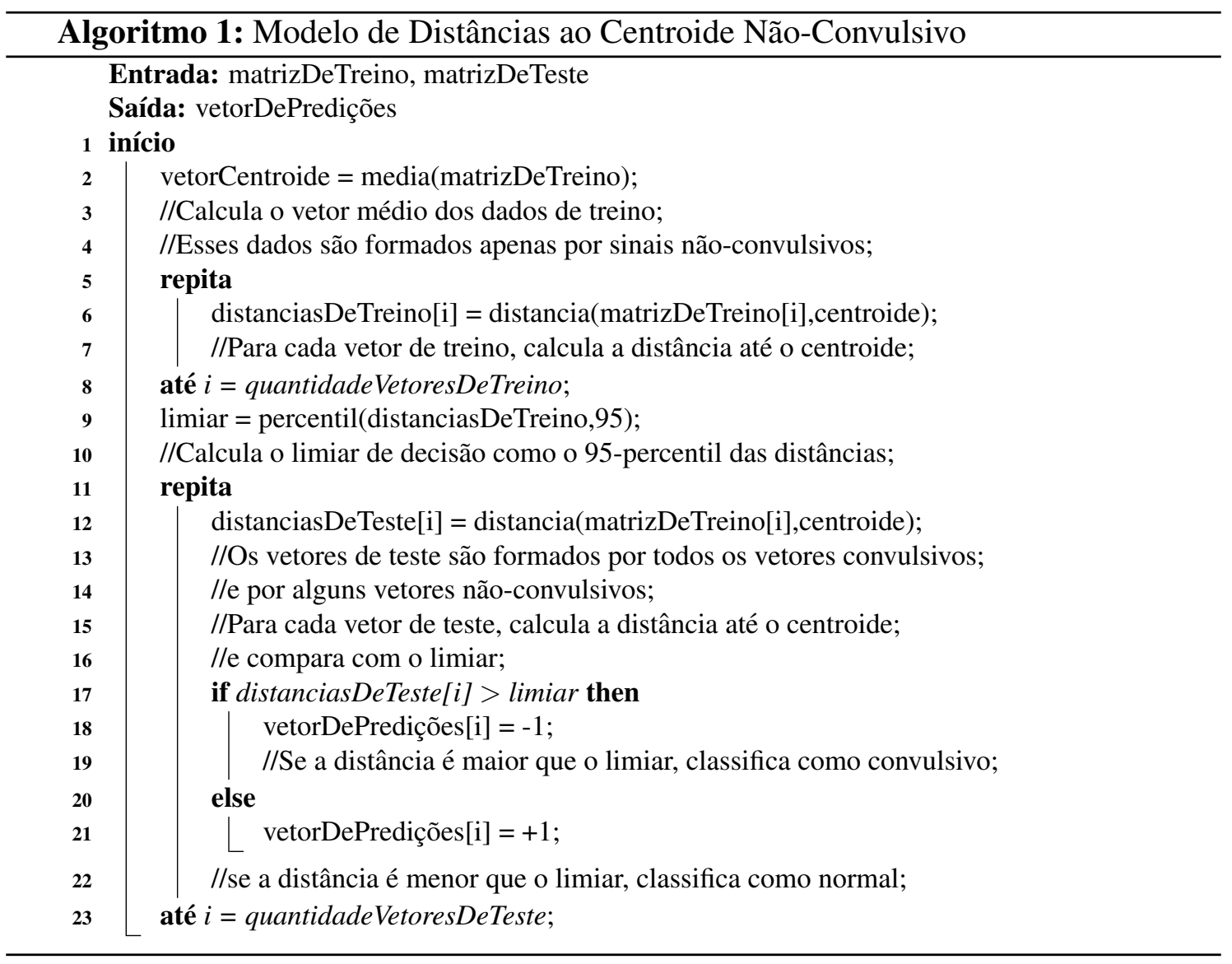

\subsection{Modelo das Distâncias Ao Centroide Convulsivo (DCC)}

O Modelo das Distâncias Ao Centroide Convulsivo (DCC) tem uma abordagem similar ao modelo anterior, porém utiliza apenas os dados convulsivos para modelar o comportamento esperado, calculando o centroide convulsivo e identificando os pontos que estejam muito distantes como não pertencentes ao conjunto de dados. Ou seja, treina-se o modelo utilizando os vetores convulsivos, calculando o limiar como o 95-percentil das distâncias ao centroide convulsivo e classificando como não-convulsivos os vetores cuja distância ao centróide está acima deste limiar. Conceitualmente, pode-se entender esse modelo como uma detecção de novidades. $\mathrm{O}$ algoritmo desse modelo segue passos idênticos ao Algoritmo 1, com duas importantes diferenças: os dados de treino são formados exclusivamente por dados convulsivos e o critério de classificação é invertido: as distâncias abaixo do limiar são classificadas como dados convulsivos e as acimas do limiar como não-convulsivos. 


\subsection{Modelo da Distâncias aos Centroides de K-Clusters (DCKC)}

Métodos baseados na distância ao centroide têm bom desempenho na identificação de anomalias aleatoriamente distribuídas em torno da massa principal de dados. Entretanto, quando as anomalias estão concentradas, ou seja, se apresentam na forma de um ou mais agrupamentos localizados regiões específica, a distância ao centroide não os detecta satisfatoriamente. Visando tornar a detecção mais robusta pode-se utilizar técnicas de agrupamento (clustering). Essas técnicas comumente são desenvolvidas a partir da suposição de que instâncias normais dos dados estão â pequenas distâncias do centroide do cluster mais próximo, enquanto anomalias estão a grandes distâncias do centroide do cluster mais próximo.

O Modelo da Distâncias aos Centroides de K-Clusters (DCKC) utiliza uma abordagem de agrupamento. Considerando os dados não-convulsivos, define-se uma quantidade $k$ de clusters, cujos centroides são calculados através dos algoritmo K-Médias [MacQueen 1967]. A quantidade ótima de clusters é estimada através do índice DaviesBouldin [Davies and Bouldin 1979]. A menor distância de cada observação aos centroides é calculada, formando um vetor de distâncias a partir do qual pode ser escolhido o limiar de decisão.

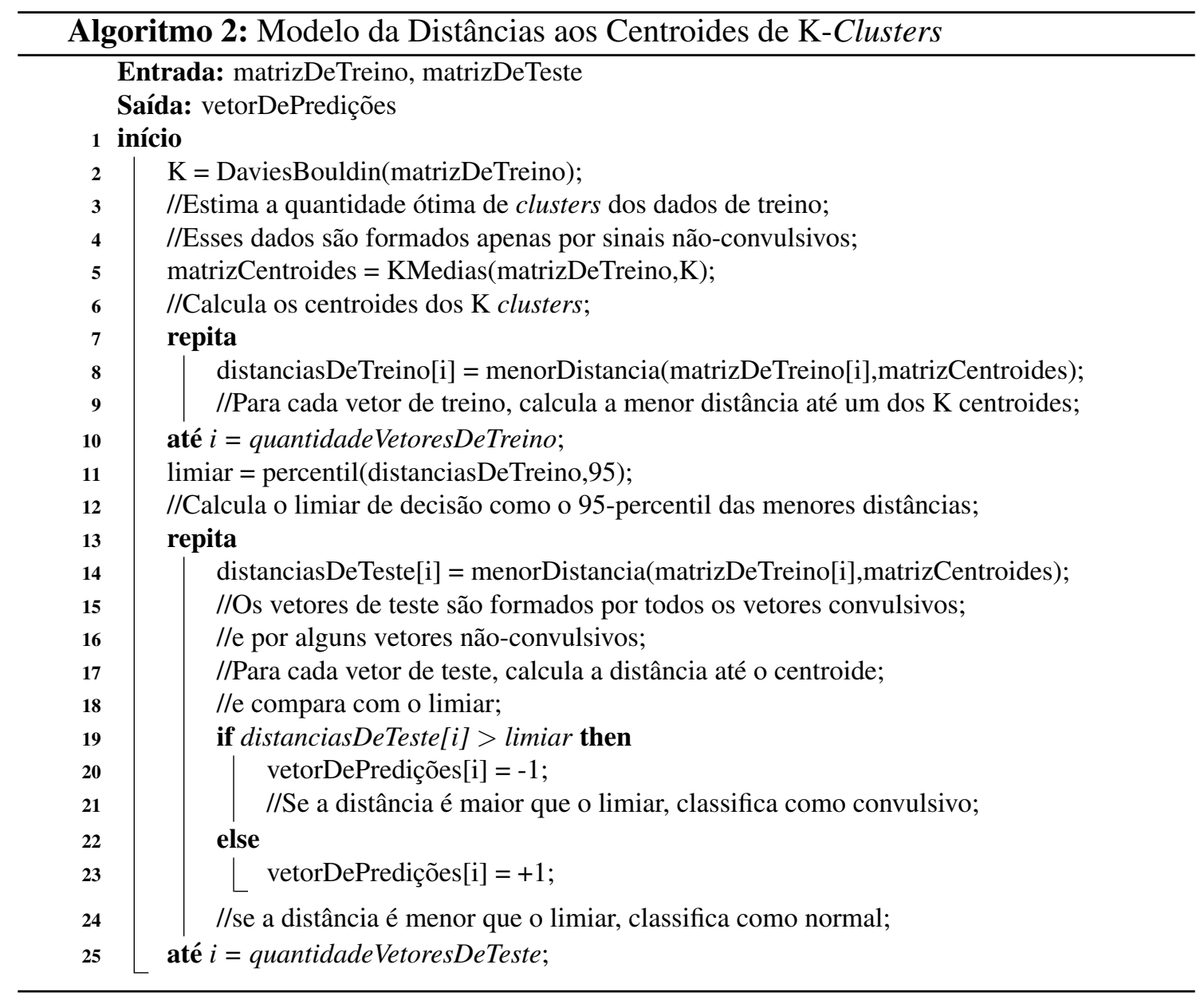

\section{Avaliação Experimental}

Neste seção, serão discutidos todos os detalhes relacionados aos dados e configurações utilizados nos experimentos realizados. Os algoritmos propostos foram implementados 
em $M A T L A B \cap$ e estão disponibilizados, assim como os dados pré-processados, no repositório: https://github.com/cabrau/anomaly-detection-eeg.

\subsection{Dados Utilizados}

O conjunto de dados utilizados neste trabalho é o mesmo utilizado nos trabalhos relacionados e consiste em gravações contínuas do EEG de escalpo, realizados em 24 pacientes pediátricos do Hospital Infantil de Boston, após a retirada da medicação para a avaliação de cirurgia de epilepsia. Os arquivos utilizados pertencem ao projeto CHBMIT Scalp EEG Database e podem ser acessados através do website: https : / / www . physionet.org/pn6/chbmit/.

Cada exame EEG apresenta uma taxa de amostragem de $256 \mathrm{~Hz}$, utilizando-se 23 canais, com duração aproximada de 1 hora. Contudo, existem gravações de até quatro horas, dependendo do quadro de crises epiléticas do paciente. Foram realizados vários exames em cada paciente, gerando um total de 686 arquivos (aproximadamente 32Gb de dados). Os arquivos estão catalogados em com convulsões e sem convulsões, onde os momentos em que ocorrem crises foram previamente classificados por especialistas. Em todos os arquivos, foram detectados 197 convulsões distribuídas em 141 arquivos, totalizando um total de 195,5 minutos para todos os pacientes.

\subsection{Extração de Atributos}

O EEG é composto de uma ampla faixa de componentes de frequência onde se destacam ritmos cerebrais associados a certos estados do cérebro. Portanto, atributos que descrevam as frequências do sinal são significativos para a identificação de crises epilépticas [Sanei and Chambers 2007]. Nesse trabalho, optamos por utilizar como atributos a Densidade Espectral de Potência do Sinal (PSD)[Hayes 1996], uma função que descreve como a energia de um sinal se distribui em suas frequências. Esse método de extração de atributos é comumente utilizado na literatura com bons resultados. Sabe-se que o sinal EEG é não-estacionário, portanto seu espectro muda com o tempo. Tal sinal pode ser aproximado como estacionário por partes, ou seja, uma sequência de segmentos de sinal estacionários independentes. Pode-se assumir que a duração de um intervalo estacionário mínimo seja de 2 segundos [McEwen and Anderson 1975]. Durante o desenvolvimento deste trabalho, também foi experimentada uma técnica de extração de atributos no domínio do tempo, através da estimação de coeficientes de Codificação Linear Preditiva (LPC) [O’Shaughnessy 1988]. Este método utiliza um modelo autorregressivo para parametrizar o sinal, e utiliza os coeficientes como atributos. Destaca-se como caracterıstica fundamental de um processo autorregressivo o fato da observação atual estar correlacionada com a observação anterior, ou seja, assume-se uma correlação significativa entre as observações anteriores, o que é uma suposição forte. Para os métodos de detecção propostos, essa técnica de extração de atributos resultou em um baixo desempenho, com acurácia abaixo de 50\%, e não serão apresentados.

\subsection{Construindo os Vetores de Atributos}

$\mathrm{Na}$ base de dados utilizada, o EEG foi captado utilizando 23 canais. Portanto, cada exame possui 23 sinais, amostrados com uma frequência de $256 \mathrm{~Hz}$. Para cada canal de EEG, vetores de atributos PSD são extraídos em janelas de tempo (épocas) de 2 segundos, intervalo no qual o sinal é considerado aproximadamente estacionário. Portanto, dada a taxa de amostragem de $256 \mathrm{~Hz}$, cada época contém 521 amostras. 
Para extrair os atributos PSD, foram realizados os seguintes passos:

- Passo 1 - Para a época corrente (janela de 2 segundos), aplicamos o método de periodograma de Welch, utilizando uma janela Gaussiana de tamanho 128. Esse procedimento foi repetido para todos os 23 canais.

- Passo 2 - Aplicamos uma escala logarítmica aos valores PSD resultantes para convertê-los em decibéis (dB). Ou seja, $P S D(d B)=10 \log _{10}(P S D)$.

- Passo 3 - Segmentamos os valores PSD (em dB) em 8 bandas de frequência, indo de $0.5 \mathrm{~Hz}$ a $25 \mathrm{~Hz}$, e calculamos a média de cada banda. Para cada canal $k$, esse procedimento leva à computação de 8 atributos $x_{1, k}, x_{1, k}, \ldots, x_{M, k}$. Esses atributos são correspondentes a energia distribuída nos principais ritmos cerebrais.

- Passo 4 - Para cada época $t$, concatenou-se as 8 médias espectrais extraídas de cada um dos 23 canais. Esse processo forma um vetor de atributos $X_{t}$ com dimensão $M \times N=184$, definido como

$$
X_{T}=\left[x_{1,1}, x_{2,1}, \ldots, x_{M, 1}|\ldots| x_{1, N}, x_{2, N}, \ldots, x_{M, N}\right]^{T}
$$

Esse procedimento foi realizado para todos os sinais de um determinado paciente. Assim, para cada paciente, temos uma matriz de atributos. Em seguida, os dados correspondentes às crises convulsivas, já previamente classificados por especialistas, foram separados, produzindo assim duas matrizes de atributos: uma com os dados referentes às crises convulsivas e outra com os dados não-convulsivos. Em geral, a quantidade instâncias na matriz com dados convulsivos corresponde a menos de $1 \%$ da quantidade de instâncias não convulsivas, evidenciando o grande desbalanceamento entre as classes.

\subsection{Métricas de Desempenho}

Tratando-se de um problema de classificação binária, utilizamos como métricas de desempenho a acurácia (AC), sensibilidade (SB) e a especificidade (EP). Estas métricas são amplamente utilizadas na literatura consultada e permitem uma comparação de resultados.

- acurácia $(A C)$ : a proporção de predições corretas, sem levar em consideração o que é positivo e o que é negativo. Esta medida é altamente suscetível a desbalanceamentos do conjunto de dados e pode facilmente induzir a uma conclusão errada sobre o desempenho do sistema:

$$
A C=\frac{\left(V_{P}+V_{N}\right)}{T O T A L}
$$

- sensibilidade $(S B)$ : a proporção de verdadeiros positivos, ou seja, a capacidade do sistema em predizer corretamente a condição para casos que realmente a têm;

$$
S B=\frac{V_{P}}{\left(V_{P}+F_{N}\right)}
$$

- especificidade $(E P)$ : A proporção de verdadeiros negativos, ou seja, a capacidade do sistema em predizer corretamente a ausência da condição para casos que realmente não a têm.

$$
E P=\frac{V_{N}}{\left(V_{N}+F_{P}\right)}
$$


É importante ressaltar que devido ao alto desbalanceamento das classes, a acurácia por si não representa uma métrica de desempenho significativa, visto que existem poucos dados convulsivos. Considerando apenas a acurácia, um classificador cuja todas predições fossem não-convulsivas ainda teria um desempenho alto, mesmo errando todas as crises convulsivas. Considerando a aplicação desejada, que é auxiliar neurologistas na detecção de crises em longos exames, o custo de um falso-negativo é maior do que o de um falsopositivo, pois o especialista poderia identificar e descartar o último, mas um falso-negativo poderia passar despercebido. Logo, a sensibilidade é uma métrica muito significativa.

\section{Resultados}

Esta seção tem por finalidade avaliar o desempenho dos três métodos proposto. Para isso, os métodos concebidos foram aplicados em três pacientes distintos, 01, 05 e 08 , onde o critério de seleção foram os resultados encontrados na literatura para cada paciente, buscando, assim, criar um subconjunto com diversidade de padrões de EEG. Na literatura, a análise dos sinais do paciente 01 possui, em geral, bom desempenho. O paciente 05 apresenta desempenho intermediário e o paciente 08 quase sempre apresenta o pior desempenho. Foram realizadas 20 rodadas de treino e teste para cada um dos três pacientes, gerando as métricas de desempenho. Em cada rodada os dados de treino e teste foram escolhidos aleatoriamente. Ao final, para cada paciente e para cada uma das três métricas utilizadas (AC, SB e EP), registramos o melhor valor, o pior valor e a média dos resultados obtidos nas 20 rodadas de treino e teste.

\subsection{Método de Distância ao Centroide Não-Convulsivo}

Para este método, na fase de treinamento, foram utilizadas $98 \%$ das instâncias nãoconvulsivas, gerando um modelo que representa os dados "normais", ou seja, sem convulsão. Já na fase de teste, utilizamos os $2 \%$ restantes das instâncias não-convulsivas e $100 \%$ das instâncias convulsivas (o que corresponde a cerca de 1\% do total de instâncias). Esta abordagem foi aplicada para cada paciente. Assim, criamos um modelo preditivo distinto para cada paciente. Para o paciente 1, por exemplo, 60420 instâncias nãoconvulsivas foram utilizadas na fase de treinamento. Já na fase de teste, foram utilizadas 611 instâncias não-convulsivas e 199 instâncias convulsivas. Assim, na fase de teste foram utilizadas ao todo 810 instâncias.

A Tabela 1 apresenta os resultados obtidos pelo Método da Distância ao Centroide Não-Convulsivo. Para cada paciente, destacamos o melhor valor obtido, o pior valor encontrado e a média dos resultados para cada uma das três métricas selecionadas (AC, SB e EP).

Tabela 1. Resultados do teste de desempenho para o Método de Distância ao Centroide Não-Convulsivo.

\begin{tabular}{|c|c|c|c|c|c|c|c|c|c|}
\hline & \multicolumn{3}{|c|}{ Paciente 1 } & \multicolumn{3}{c|}{ Paciente 5 } & \multicolumn{3}{c|}{ Paciente 8 } \\
\cline { 2 - 11 } & Melhor & Pior & Média & Melhor & Pior & Média & Melhor & Pior & Média \\
\hline AC & 96.39 & 93.63 & 95.37 & 97.10 & 93.57 & 95.26 & 95.72 & 92.43 & 94.10 \\
\hline SB & 96.88 & 96.88 & 96.88 & 97.26 & 97.26 & 97.26 & 92.68 & 91.87 & 92.36 \\
\hline EP & 96.31 & 93.12 & 95.14 & 97.07 & 92.91 & 94.90 & 98.34 & 92.27 & 95.28 \\
\hline
\end{tabular}


Observa-se que, apesar de considerar uma modelagem simples da distribuição dos dados, o modelo gerou bons resultados utilizando os atributos PSD. Portanto, a distribuição de energia entre as frequências do sinal segue a hipótese inicial assumida de que os dados se distribuem ao redor do centroide. De fato, os ritmos cerebrais de uma crise convulsiva são compostos por frequências que, em geral, são distinguíveis do comportamento normal.

Observa-se também que os resultados do paciente 8 apresentam um desempenho ligeiramente inferior ao dos demais pacientes. Possivelmente este resultado deve-se ao tipo de crise epiléptica do paciente 08 ter um padrão similar ao padrão normal. De fato, nos resultados encontrados na literatura o paciente 08 obtém sempre o pior desempenho. No trabalho de Liang et al. 2019, por exemplo, o paciente 8 atingiu sensibilidade de $60 \%$. Encontra-se na literatura médica casos onde não há alteração perceptível no EEG durante crises, dificultando tanto o diagnóstico quanto a detecção por algoritmos. Uma vez que trata-se de uma condição onde os padrões podem variar enormemente entre pacientes, esse tipo de caso pode ocorrer. Neste cenário, apesar de inferior aos pacientes 1 e 5 , as médias do paciente 8 ficaram sempre acima de $90 \%$, indicando a robustez desse método mesmo para um caso mais complexo.

\subsection{Método de Distância ao Centroide Convulsivo}

Com a finalidade de avaliar o Método de Distância ao Centroide Convulsivo, 70\% dos dados convulsivos foram selecionados aleatoriamente para formar o modelo, na fase de treinamento. Já na fase de teste foram utilizados os $30 \%$ restantes dos dados convulsivos juntamente com $1 \%$ dos dados não convulsivos. Essa proporção difere consideravelmente do método anterior devido a diferença entre as quantidades de dados de crises disponíveis.

A Tabela 2 apresenta os resultados obtidos pelo Método da Distância ao Centroide Convulsivo. Para cada paciente, destacamos o melhor valor obtido, o pior valor encontrado e a média dos resultados para cada uma das três métricas selecionadas (AC, SB e $\mathrm{EP})$.

Tabela 2. Resultados do teste de desempenho para o Método de Distâncias ao Centroide Convulsivo.

\begin{tabular}{|c|c|c|c|c|c|c|c|c|c|}
\hline & \multicolumn{3}{|c|}{ Paciente 1 } & \multicolumn{3}{c|}{ Paciente 5 } & \multicolumn{3}{c|}{ Paciente 8 } \\
\cline { 2 - 10 } & Melhor & Pior & Média & Melhor & Pior & Média & Melhor & Pior & Média \\
\hline AC & 100 & 98.66 & 99.44 & 99.12 & 93.83 & 96.06 & 97.03 & 69.64 & 93.83 \\
\hline SB & 100 & 90.00 & 97.00 & 100 & 86.36 & 97.05 & 94.31 & 91.87 & 92.54 \\
\hline EP & 100 & 99.02 & 99.68 & 99.51 & 93.17 & 95.95 & 100 & 52.78 & 94.72 \\
\hline
\end{tabular}

Observa-se que esse modelo consegue altas taxas de acerto nos melhores casos, conseguindo até $100 \%$ no paciente 1 , mas também apresenta uma maior variância. Os piores casos do paciente 8 apresentam valores bastante inferiores em relação aos cenários apresentados anteriormente, que obtiveram resultados mais consistentes. Novamente, é importante salientar que o EEG de cada paciente possui características únicas, o que torna métodos de reconhecimento de padrões nesse sinal altamente dependentes do paciente. Em comparação com o modelo DCNC, o modelo DCC é treinado com uma quantidade consideravelmente inferior de dados, uma vez que existem menos dados convulsivos dis- 
poníveis, o que dificulta a generalização da classificação e pode explicar o seu desempenho inferior nos piores casos.

\subsection{Método de Distâncias aos Centroides de K-Clusters}

Por fim, avaliamos o Método de Distâncias aos Centroides de K-Clusters, no qual são calculadas as menores distâncias aos centroides de uma quantidade ótima de clusters envolvendo os dados não-convulsivos. Assim como no experimento com o modelo DCNC, foram utilizadas $98 \%$ das instâncias não-convulsivas para treino. O conjunto de teste é formado por $2 \%$ das instâncias não-convulsivas e por todas as instâncias convulsivas de cada paciente.

Pelo índice Davies-Bouldin, a quantidade ótima de clusters encontrada foi 3. A Tabela 3 apresenta os resultados obtidos pelo Método de Distâncias aos Centroides de KClusters. Para cada paciente, destacamos o melhor valor obtido, o pior valor encontrado e a média dos resultados para cada uma das três métricas selecionadas (AC, SB e EP).

Tabela 3. Resultados do Método de Distâncias aos Centroides de K-Clusters

\begin{tabular}{|c|c|c|c|c|c|c|c|c|c|}
\hline & \multicolumn{3}{|c|}{ Paciente 1 } & \multicolumn{3}{c|}{ Paciente 5 } & \multicolumn{3}{c|}{ Paciente 8 } \\
\cline { 2 - 11 } & Melhor & Pior & Média & Melhor & Pior & Média & Melhor & Pior & Média \\
\hline AC & 97.03 & 93.42 & 94.98 & 96.68 & 93.57 & 94.77 & 98.03 & 94.41 & 96.15 \\
\hline SB & 96.88 & 90.63 & 96.56 & 95.89 & 94.52 & 95.75 & 97.56 & 92.68 & 97.32 \\
\hline EP & 97.05 & 93.12 & 94.73 & 97.07 & 93.15 & 94.60 & 98.34 & 92.82 & 95.36 \\
\hline
\end{tabular}

Esse método obteve médias bastante elevadas, próximas ou superiores aos resultados dos métodos apresentados anteriormente. Além disso, o Método de Distâncias aos Centroides de K-Clusters manteve uma consistência de resultados entre os pacientes. No caso do paciente 8 , ele obteve os melhores resultados. De fato, a vantagem desse método é que ele utiliza uma representação mais fiel da distribuição dos dados, considerando vários centroides ao invés de um, sendo, portanto, mais robusto a ruídos. Porém, esse método tem como desvantagem o alto custo computacional em relação aos anteriores, principalmente na etapa de treino, onde é preciso executar o algoritmo K-Médias diversas vezes para um grande volume de dados. Adicionalmente, a etapa de teste também apresenta um custo maior, uma vez que é preciso testar a distância de cada instância a vários centroides ao invés de somente um.

\subsection{Análise Comparativa}

Por fim, realizamos uma análise comparativa entre os três modelos propostos neste artigo e as abordagens encontradas na literatura que utilizaram a mesma base de dados e as mesmas métricas de desempenho. A Tabela 4 ilustra a média dos valores obtidos para as três métricas selecionadas (AC, SB e EP), para cada um dos métodos analisados. A coluna Tipo indica se o classificador foi treinado e testado para cada paciente separadamente (paciente específico), como realizado neste trabalho, ou foi treinado e testado com os dados de todos os pacientes (inter-pacientes, IP) em conjunto.

De forma geral, os três modelos propostos obtiveram bons resultados, dentro das condições estabelecidas. Apesar de serem utilizados métodos relativamente simples, o desempenho foi comparável aos melhores resultados do estado da arte, que utilizam técnicas 
Tabela 4. Análise Comparativa

\begin{tabular}{llllll}
\hline Autor, Ano & Classificador & Tipo & AC \% & SB \% & EP \% \\
\hline [Shoeb and Guttag 2010] & SVM & Paciente-específico & 96 & - & - \\
[Acharya et al. 2011] & SVM & Paciente-específico & 96 & 96 & 97 \\
[Fergus et al. 2016] & KNNC & Inter-pacientes & 88 & 88 & 93 \\
[Bhattacharyya and Pachori 2017] & Random Forest & Paciente-específico & 94.4 & 97.9 & 99.5 \\
[Liang et al. 2019] & LTCN & Inter-pacientes & 84 & 99 & 99 \\
Neste trabalho & DCNC & Paciente-específico & 95.3 & 96.1 & 95 \\
Neste trabalho & DCC & Paciente-específico & 96.4 & 95.5 & 96.7 \\
Neste trabalho & DCKC & Paciente-específico & 95.3 & 96.5 & 96.6 \\
\hline
\end{tabular}

de classificação mais sofisticadas baseadas em aprendizado supervisionado. Isso demonstra a relevância da abordagem de detecção de anomalias no problema de identificação de crises convulsivas em EEG.

\section{Conclusões e Trabalhos Futuros}

A principal contribuição desse trabalho foi a concepção e avaliação de três modelos distintos para identificar crises convulsivas, utilizando detecção de anomalias e atributos de frequência. Não encontramos na literatura nenhum trabalho que tenha investigado essa extratégia. Os resultados experimentais mostraram que os modelos propostos apresentam um desempenho semelhante aos melhores resultados encontrados na literatura. Além disso, os modelos propostos possuem robustez para lidar com sinais ruidosos, simplicidade de treino e baixo custo computacional. Como trabalhos futuros, pretendemos investigar a aplicação destes métodos na classificação inter-pacientes.

\section{Agradecimentos}

Os autores agradecem ao Conselho Nacional de Desenvolvimento Científico e Tecnológico (CNPq) pelo apoio financeiro concedido na forma de bolsa de mestrado que tornou este trabalho possível.

\section{Referências}

[A. Zimek and Kriegel 2012] A. Zimek, E. S. and Kriegel, H. (2012). A survey on unsupervised outlier detection in high-dimensional numerical data. Statistical Analysis and Data Mining: The ASA Data Science Journal, 5(5):363-387.

[Acharya et al. 2011] Acharya, U. R., Sree, S. V., Chattopadhyay, S., YU, W., and ANG, P. C. A. (2011). Application of recurrence quantification analysis for the automated identification of epileptic eeg signals. International Journal of Neural Systems, 21(03):199-211. PMID: 21656923.

[Adeli and Ghosh-Dastidar 2010] Adeli, H. and Ghosh-Dastidar, S. (2010). AUTOMATED EEG-BASED DIAGNOSIS OF NEUROLOGICAL DISORDERS: Inventing the Future of Neurology. New York: CRC Press.

[Bhattacharyya and Pachori 2017] Bhattacharyya, A. and Pachori, R. B. (2017). A multivariate approach for patient-specific eeg seizure detection using empirical wavelet transform. IEEE Transactions on Biomedical Engineering, 64(9):2003-2015. 
[Chan et al. 2008] Chan, A. M., Sun, F. T., Boto, E. H., and Wingeier, B. M. (2008). Automated Seizure onset detection for accurate onset time determination in intracranial EEG. Clinical Neurophysiology, 119. pp. 2687-2696.

[Davies and Bouldin 1979] Davies, D. L. and Bouldin, D. W. (1979). A cluster separation measure. IEEE Transactions on Pattern Analysis and Machine Intelligence, PAMI$1(2): 224-227$.

[Fergus et al. 2016] Fergus, P., Hussain, A., Hignett, D., Al-Jumeily, D., Abdel-Aziz, K., and Hamdan, H. (2016). A machine learning system for automated whole-brain seizure detection. Applied Computing and Informatics, 12(1):70 - 89.

[Hayes 1996] Hayes, M. H. (1996). Statistical Digital Signal Processing and Modeling. USA: John Wiley and Sons.

[Kanashiro 2006] Kanashiro, A. L. A. N. (2006). EPILEPSIA: prevalência, características epidemiológicas e lacuna de tratamento farmacológico. . 2006. $135 \mathrm{f}$. Master's thesis, Tese (Faculdade de Ciências Médicas da Universidade Estadual de Campinas).

[Khan et al. 2012] Khan, Y. U., Rafiuddin, N., and Farooq, O. (2012). Automated seizure detection in scalp eeg using multiple wavelet scales. In 2012 IEEE International Conference on Signal Processing, Computing and Control, pages 1-5.

[Knorr 2000] Knorr, E.; NG, R. T. T. V. (2000). Distance-based outliers: Algorithms and applications. VLDB Journal, 8(3):237-253.

[Liang et al. 2019] Liang, W., Pei, H., Cai, Q., and Wang, Y. (2019). Scalp eeg epileptogenic zone recognition and localization based on long-term recurrent convolutional network. Neurocomputing.

[MacQueen 1967] MacQueen, J. (1967). Some methods for classification and analysis of multivariate observations. In Proc. Fifth Berkeley Symp. Math. Stat. Probab. Vol. 1 Stat., pages 281-297, Berkeley, Calif. University of California Press.

[McEwen and Anderson 1975] McEwen, J. A. and Anderson, G. B. (1975). Modeling the stationarity and gaussianity of spontaneous electroencephalographic activity. IEEE Transactions on Biomedical Engineering, BME-22(5):361-369.

[Niedermeyer and da Silva 2001] Niedermeyer, E. and da Silva, F. L. (2001). Electroencephalography - Basic Principles, Clinical Applications and Related Fields, volume 1. Williams Williams.

[O'Shaughnessy 1988] O'Shaughnessy, D. (1988). Linear predictive coding. IEEE Potentials, 7(1):29-32.

[Sanei and Chambers 2007] Sanei, S. and Chambers, J. A. (2007). EEG Signal Processing. England: John Wiley and Sons.

[Shoeb and Guttag 2010] Shoeb, A. and Guttag, J. (2010). Application of Machine Learning To Epileptic Seizure Detection. Appearing in Proceedings of the 27th International Conference on Machine Learning , Haifa, Israel.

[Subasi and Ercebeli 2005] Subasi, A. and Ercebeli, E. (2005). Classification of EEG signal using neural network an logistic regression. Computer Methodis and Programs in Biomedicine, 78. pp. 87-99.

[Varun Chandola 2009] Varun Chandola, Arindam Banerjee, V. K. (2009). Anomaly detection: A survey. ACM Computing Surveys (CSUR), 41(3).

[Webb 2002] Webb, A. (2002). Statistical Pattern Recognition. John Wiley Sons.

[World Health Organization 2017] World Health Organization (2017). Epilepsy fact sheet. 